TITLE:

\title{
Coulomb Effect in Nucleon-Deuteron Elastic Scattering
}

\author{
$\operatorname{AUTHOR}(\mathrm{S})$ :
}

Fukukawa, Kenji; Fujiwara, Yoshikazu

\section{CITATION:}

Fukukawa, Kenji ...[et al]. Coulomb Effect in Nucleon-Deuteron Elastic Scattering. FewBody Systems 2013, 7-10: 1331-1334

ISSUE DATE:

2013-01

URL:

http://hdl.handle.net/2433/170258

\section{RIGHT:}

The final publication is available at www.springerlink.com; この論文は 出版社版でありません。引用の際には出版社版をご確認ご利用くださ $\omega_{\circ}$; This is not the published version. Please cite only the published version. 
Few-Body Systems (FB20) manuscript No.

(will be inserted by the editor)

\title{
Coulomb effect in Nucleon-Deuteron Elastic Scattering
}

\author{
K. Fukukawa · Y. Fujiwara
}

Received: date / Accepted: date

\begin{abstract}
We have applied the quark-model nucleon-nucleon interaction fss 2 to the nucleon-deuteron scattering. The screened cutoff Coulomb force in the momentum space has been incorporated to the proton-deuteron scattering by extending the matching condition, which is proposed by Vincent and Phatak for the sharply cutoff Coulomb problem. The model fss 2 can reproduce the overall characteristics of the experimental data. It is found that fss 2 partially incorporates the attractive feature, which accords with the good reproduction of the triton binding energy and neutron-deuteron doublet scattering length. This attractive feature is due to the off-shell effect related to the quark-model description of the short-range repulsion of the two-nucleon force.
\end{abstract}

Keywords Quark-model nucleon-nucleon interaction · Nucleon-deuteron scattering

\section{Introduction}

The three-nucleon $(3 N)$ system is appropriate to study the nucleon-nucleon $(N N)$ interaction since many techniques for rigorous calculations have been developed. The quark-model (QM) baryon-baryon interaction is constructed in the framework of the resonating-group method for two three-quark clusters. The short-range repulsion of the $N N$ interaction is mainly described by the nonlocal quark-exchange kernel, which gives quite different off-shell properties from standard meson-exchange potentials. The deficiency of the triton binding energy by our QM $N N$ interaction fss2 [1] is about

Presented at the 20th International IUPAP Conference on Few-Body Problems in Physics, 20 - 25 August, 2012, Fukuoka, Japan

K. Fukukawa

RIKEN Nishina Center, 2-1 Hirosawa, Wako, Saitama 351-0198, Japan,

Tel.: +81-48-462-1572

Fax: +81-48-462-1569

E-mail: fukukawa@riken.jp

Y. Fujiwara

Department of Physics, Kyoto University, Kitashirakawa Oiwakecho, Sakyo-ku, Kyoto 6068502, Japan 

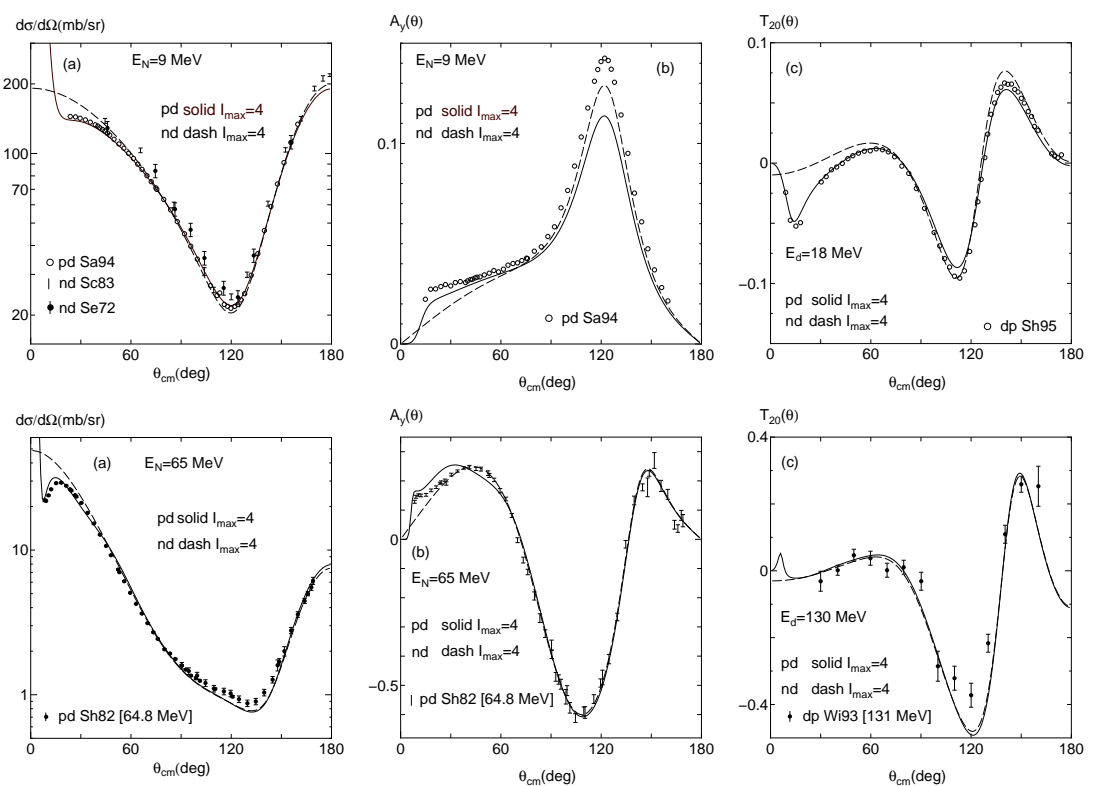

Fig. $1 N d$ elastic scattering observables at $E_{N}=9$ (upper panels) and $65 \mathrm{MeV}$ (lower panels), compared with the experimental data; (a) the elastic differential cross section $d \sigma / d \Omega$, (b) the nucleon vector analyzing power $A_{y}(\theta)$, and (c) the deuteron tensor analyzing power $T_{20}(\theta)$. The solid curves show the results of the $p d$ calculations using the Coulomb cutoff radius $\rho=8$ $\mathrm{fm}$, and the dashed curves show $n d$ predictions. The experimental data. are taken from Refs. [2] for Sa94, [3] for Sc83, [4] for Se72, [5] for Sh95, [6] for Sh82 and [7] for Wi93.

$350 \mathrm{keV}$ [8], which is far smaller than $0.5-1 \mathrm{MeV}$, predicted by standard mesonexchange potentials. It is therefore interesting to examine the nonlocal effect of QM $N N$ interaction on the $3 N$ scattering observables.

The Coulomb force should be taken into account for the comparison with ample and precise experimental proton-deuteron $(p d)$ scattering data. The incorporation of the Coulomb force to the three-body scattering problem is a challenging task. In recent years, some development have been made based on the Kohn variational approach [9] and on the coodinate space Faddeev integral equations [10,11]. The standard treatment in the momentum space is the screening and renormalization approach [12-14]. In this contribution, a newly-developed method in Ref. [15] based on the Vincent-Phatak method [16] is successfully applied to the $p d$ elastic scattering and we examine the off-shell effect of the QM $N N$ interaction.

\section{Results}

In applying fss 2 to the nucleon-deuteron $(N d)$ elastic scattering in the Faddeev formalism for composite particles, the Alt-Grassberger-Sandhas equations [17] are solved in the momentum space. The channel-spin formalism is used. We introduce the $N N$ interaction up to the total angular momentum $I_{\max }=4$. The charge independence 


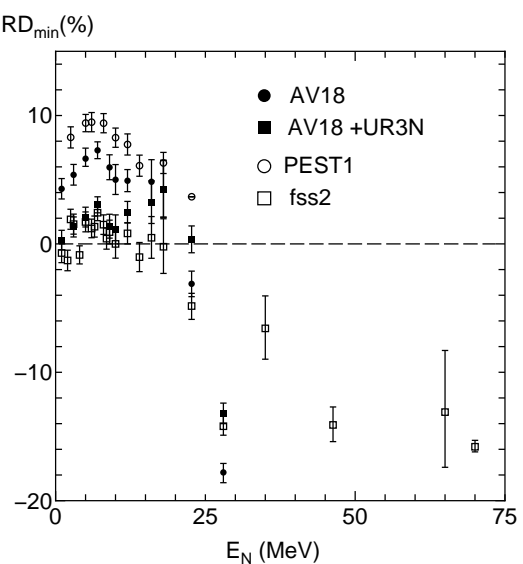

Fig. 2 Relative deviation of the $p d$ differential cross section minima from the experimental data. The experimental data are taken from [2]. The AV18 (filled circles) and AV18+URIX $3 N$ results (filled squares) are from Kievsky et al. [9] and the results using the PEST1 potential (open circles) are from Alt et al [12]. breaking and charge symmetry breaking are not included, nor the $3 N$ isospin $T=3 / 2$ component for the $p d$ scattering. Our calculations do not introduce $3 N$ forces.

The sharply cutoff Coulomb force is introduced at the quark level, which leads to the error-function-type Coulomb force between two protons. The screened Coulomb force between the proton and the deuteron is obtained by folding the $p p$ Coulomb potential with the realistic deuteron wave function. We calculate the $N N$-matrix for isospin 1 channel using $t_{\text {eff }}^{I=1}=(2 / 3) t_{p p}+(1 / 3) t_{n p}$ [18]. In Ref. [15], we propose a practical method of solving the screened Coulomb problem by extending the Vincent-Phatak method for the sharply cutoff Coulomb force [16]. We can extract the nuclear phase shift by imposing the matching condition for the asymptotic wave functions.

We have examined the $N d$ elastic scattering observables up to $E_{N}=65 \mathrm{MeV}$. Some examples are shown in Fig. 1. The overall characteristic of the experimental data is reproduced, while discrepancies at the maximum points of the vector analyzing power (so-called " $A_{y}$ puzzle") still exist. The Coulomb effect is important at all angles in the low-energy region, but confined to forward angles in the higher energy region.

The energy dependence of the minimum points of the elastic differential cross sections (diffraction minima) is often discussed as Sagara discrepancy [2] and is very important to discuss the $3 N$ force effect. In order to examine the energy dependence of the diffraction minima quantitatively, we show in Fig. 2 the relative differences, which are defined by $\mathrm{RD}_{\min }=\left[\left(\frac{d \sigma}{d \Omega}\right)_{\text {cal. }}\left(\theta_{\min }\right)-\left(\frac{d \sigma}{d \Omega}\right)_{\text {exp. }}\left(\theta_{\min }\right)\right] /\left(\frac{d \sigma}{d \Omega}\right)_{\text {exp. }}\left(\theta_{\min }\right)$ in the energy region $E_{p}<70 \mathrm{MeV}$. On the low-energy side $E_{p}<18 \mathrm{MeV}$, the minimum values of the $p d$ differential cross sections calculated by fss 2 reproduce the experimental data at accuracy less than $2 \%$. In Ref. [9], the Urbana IX (URIX) $3 N$ potential lessens the cross sections at the diffraction minima, resulting in a good agreement with the experimental data. In our result, fss 2 takes into account this attractive effect at the $N N$ level. This result accords with the good reproduction of the triton binding energy and $n d$ doublet scattering length [19].

\section{Summary}

We have incorporated the screened Coulomb potential for the $p d$ scattering and examined elastic scattering observables up to $E_{N}=65 \mathrm{MeV}$. The QM $N N$ interaction fss 2 can reproduce the overall characteristics of the experimental data. We have found, from the analysis of the diffraction minima, that fss 2 partially incorporates the attractive effect in the doublet $S$-channel, which is usually attributed to the $3 N$ force in the standard description by meson-exchange potentials. These results reflect the off-shell effect related to the non-locality of the QM $N N$ interaction. 
Acknowledgements This work was supported by Grants-in-Aid for Scientific Research (S) and (C) from the Japan Society for the Promotion of Science (JSPS) (Grant No. 23224006 and No. 23540302, respectively), and by a Grant-in-Aid for the Global COE Program "The Next Generation of Physics, Spun from Universality and Emergence" from the Ministry of Education, Culture, Sports, Science and Technology (MEXT) of Japan. It was also supported by core-stage backup subsidies from Kyoto University. The numerical calculations were carried out on SR16000 at YITP in Kyoto University and on the high-performance computing system Intel Xeon X5680 at RCNP in Osaka University.

\section{References}

1. Fujiwara, Y., Suzuki, Y., Nakamoto, C.: Baryon-baryon interactions in the $S U_{6}$ quark model and their applications to light nuclear systems. Prog. Part. Nucl. Phys. 58, 439 (2007)

2. Sagara, K., Oguri, H., Shimizu, S., Maeda, K., Nakamura, H., Nakashima., T., Morinobu, S.: Energy dependence of analyzing power $A_{y}$ and cross section for $p+d$ scattering below $18 \mathrm{MeV}$. Phys. ReV. C50, 576 (1994)

3. Schwarz, P., Klages, H.O., Doll, P., Haesner, B, Wilczynski, J., Zeitnitz, B.: Kecskemeti, J.: Elastic neutron-deuteron scattering in the energy range from $2.5 \mathrm{MeV}$ to $30 \mathrm{MeV}$. Nucl. Phys. A398, 1 (1983)

4. Seagrave, J.D., Hopkins, J.C., Dixon, D.R., Keaton Jr, P.W., Kerr, E.C., Niiler, A., Sherman, R.H., Walter., R.K.: Elastic scattering and polarization of fast neutrons by liquid deuterium and tritium. Ann. of. Phys. 74, 250 (1972)

5. Shimizu, S., Sagara, K., Nakamura, H., Maeda, K., Miwa, T., Nishimori, N., Ueno, S., Nakashima, T., Morinobu, S.: Analyzing powers of $p+d$ scattering below the deuteron breakup threshold. Phys. Rev. C52, 1193 (1995)

6. Shimizu, H., Imai, K., Tamura, N., Nisimura, K., Hatanaka, K., Saito, T., Koike, Y., Taniguchi, Y.: Analyzing powers and cross sections in elastic $p d$ scattering at $65 \mathrm{MeV}$. Nucl. Phys. A382, 242 (1982)

7. Witała, H., Glöckle, W., Antonuk, L.E., Arvieux, J., Bachelier, D., Bonin, B., Boudard, A., Cameron, J.M., Fielding, H.W., Garçon, M., Jourdan, F., Lapointe, C., McDonald, W.J., Pasos, J., Roy, G., The, I., Tinslay, J., Tornow, W., Yonnet, J., Ziegler, W.: Complete set of deuteron analyzing powers in deuteron-proton elastic scattering: measurement and realistic potential prediction. Few-Body Systems, 15, 67 (1993)

8. Fujiwara, Y., Suzuki, Y., Kohno, M., Miyagawa, K.: Addendum to triton and hypertriton binding energies calculated from $S U_{6}$ quark-model baryon-baryon interactions. Phys. Rev. C77, 027001 (2008)

9. Kievsky, A., Viviani, M., Rosati, S.: Polarization observables in $p$ - $d$ scattering below 30 MeV. Phys. Rev. C64, 024002 (2001)

10. Ishikawa, S.: Low-Energy proton-deuteron scattering with a Coulomb-modified Faddeev equation. Few-Body Systems 32, 229 (2003)

11. Ishikawa, S.: Coordinate space proton-deuteron scattering calculations including Coulomb force effects. Phys. Rev. C80, 054002 (2009), and private communication

12. Alt, E.O., Mukhamedzhanov, A.M., Nishonov, M.M., Sattarov, A.I.: Proton-deuteron elastic scattering from 2.5 to $22.7 \mathrm{MeV}$. Phys. Rev. C65, 064613 (2002)

13. Deltuva, A., Fonseca, A.C., Sauer, P.U.: Momentum-space treatment of the Coulomb interaction in three-nucleon reactions with two protons. Phys. Rev. C71, 054005 (2005)

14. Deltuva, A., Fonseca, A.C., Sauer, P.U.: Momentum-space description of three-nucleon breakup reactions including the Coulomb interaction. Phys. Rev. C72, 054004 (2005)

15. Fujiwara, Y., Fukukawa, K.: A practical method of solving cutoff Coulomb problems in momentum space - Applications to the Lippmann-Schwinger resonating-group method and the $p d$ elastic scattering - Prog. Theor. Phys. 128, 301 (2012)

16. Vincent, C.M., Phatak, S.C.: Accurate momentum-space method for scattering by nuclear and Coulomb potentials. Phys. Rev. C10, 391 (1974)

17. Alt, E.O., Grassberger, P., Sandhas, W.: Reduction of the three-particle collision problem to multi-channel two-particle Lippmann-Schwinger equations. Nucl. Phys. B2, 167 (1967)

18. Witała, H., Glöckle, W., Kamada, H.: Charge-independence breaking in the three-nucleon system. Phys. Rev. C43, 1619 (1991)

19. Fukukawa, K., Fujiwara, Y.: Effective-range expansion of neutron-deuteron scattering studied by a quark-model nonlocal Gaussian potential. Prog. Theor. Phys. 125, 957 (2011) 